\title{
NON-LOCAL MEANS SAR DESPECKLING BASED ON SCATTERING
}

\author{
Gerardo Di Martino, Alessio Di Simone, Antonio Iodice, Daniele Riccio, Giuseppe Ruello \\ Università di Napoli Federico II, Via Claudio 21, 80125, Napoli, Italy
}

\begin{abstract}
Speckle reduction in Synthetic Aperture Radar (SAR) images is an essential pre-processing step for a correct analysis and interpretation of SAR data. This justifies the huge effort in the image processing community to develop more and more accurate despeckling techniques in order to reduce speckle effects and then improve readability of SAR imagery also for non SAR expert users. Up to now, nonlocal means approaches provide the most promising and effective despeckling performances. In this paper we develop a new non-local means despeckling technique based on electromagnetic scattering mechanisms. The proposed method, based on a physically meaningful similarity criterion for distance evaluation, is theoretically assessed, tested on a simulated SAR image, and compared to the state of the art.
\end{abstract}

Index Terms - Synthetic Aperture Radar, electromagnetic scattering, despeckling, nonlocal means

\section{INTRODUCTION}

Remote sensing data represent an essential and useful tool in Earth observation and monitoring, as well as for the study and the analysis of other celestial bodies. Thanks to their allday and all-weather capability, Synthetic Aperture Radar (SAR) systems are of great interest, as the recent ESA Sentinel-1 mission shows. However, due to speckle, SAR images interpretation is not at all an easy task, also for a SAR expert user. In order to increase the readability and facilitate the interpretation of the SAR imagery content, a despeckling pre-processing step is frequently advisable. In this way, just removing speckle effects, it is possible to dramatically broaden SAR products recipients, consequently increasing SAR systems applications. Starting from the very simple mean filter, optimum for suppressing Additive White Gaussian Noise (AWGN), a large amount of despeckling techniques has been conceived and designed [1]-[4]. Patchbased non-local techniques represent the state of the art in SAR despeckling. A unique appealing and interesting shared idea is behind these apparently different techniques: the best way (with respect to some proper metrics) to reduce speckle effects is to average only similar objects. Patch-based techniques take into account this general idea, considering an object as a fixed shape patch [2], [3]; usually they are non-local techniques. Non-local techniques show the most interesting results and performances, thanks to their appealing approach based on the concept that geometrical proximity does not necessarily imply similarity. They differ on how similarity is defined and how, eventually, patches are aggregated and combined. The state of the art is then essentially characterized by a similarity taking into account only statistical and geometrical metrics, regardless of the physics behind SAR image formation. As a consequence of this incomplete approach, two physically different objects could share the same representation in the SAR imagery and then be averaged, regardless of their differences. Therefore, it is necessary to introduce a further similarity criterion based on a new distance taking into account scattering mechanisms.

\section{NON LOCAL MEANS BASED ON SCATTERING}

In a general framework, a non-local means filter can be expressed in the following form

$$
I_{N L}(c)=\sum_{s \in \Omega} w(c, s) I(s)
$$

where $I_{N L}(c)$ is the filtered intensity image evaluated at the current pixel location $c, \Omega$ is a search window centered at $c$, $I(s)$ is the noisy intensity image evaluated in a generic pixel location $s$ of the search window and $w(c, s)$ is the weight assigned to pixels located in $s$ in the estimation of the current pixel. Weights are in general a decreasing function of the distance $d(c, s)$ between the current pixel and the generic one of the search window. In this paper we consider an exponential weight, i.e.:

$$
w(c, s)=e^{-d(c, s)} .
$$

Following the approach used in [4], we define the distance $d(c, s)$ as a linear combination of three terms:

$$
d(c, s)=\lambda_{g} d_{g}(c, s)+\lambda_{s c} d_{s c}(c, s)+\lambda_{s t} d_{s t}(c, s),
$$

where $d_{g}(c, s), d_{s c}(c, s)$ and $d_{s t}(c, s)$ represent a geometrical distance, a scattering distance and a statistical distance, 
respectively; they are properly scaled by the parameters $\lambda_{\mathrm{g}}$, $\lambda_{\text {sc }}$, and $\lambda_{\text {st }}$ respectively. The innovative term is the second one. In fact, the geometrical and statistical distances are defined as in [4]

$$
\begin{gathered}
d_{g}(c, s)=\|c-s\|^{2} \\
d_{s t}(c, s)=\log _{2}\left(\frac{1}{2}\left(\frac{i(s)}{i(c)}+\frac{i(c)}{i(s)}\right)\right),
\end{gathered}
$$

$i(\cdot)$ being the square root of intensity, namely the amplitude; the scattering distance $d_{s c}$ is defined in the following. To this aim, it is important to note that scattering from a natural surface depends upon a huge number of surface and sensor parameters, depending on the adopted surface and scattering models. Considering usual scattering and surface models, the parameters influencing scattering are the following: look angle, operating frequency, chirp bandwidth, polarization, soil dielectric constant and conductivity, local slopes and other (at least two) parameters describing surface roughness. The first four parameters describe the sensor and the remaining ones are relevant to the surface. Leaving aside the sensor parameters, scattering distance could properly take into account differences in all the surface parameters: two objects can be considered similar only if all their parameters can be regarded as similar. It is clear that this physical-based approach requires huge a priori knowledge, since, at least in principle, ground truth about all the surface parameters is needed. In order to overcome this issue, it has to be noted that scattering behavior depends in different ways on the surface parameters, i.e., a fixed relative variation of each parameter gives rise to very different scattering variations. This behavior obviously depends on the surface and scattering models used. However, the major scattering sensitivity is against local slopes. As a consequence, a simple and sufficiently accurate (for the considered purpose) method to estimate the scattering distance between two objects is to evaluate the similarity of their local slopes, or, equivalently, of their local incidence angle. This similarity criterion can be set up if a Digital Elevation Model (DEM) projected in the SAR geometry is at hand. If it is not the case, authors proposed in [5] an extremely simple method to provide a gross estimate of local slopes from a single intensity SAR image using shape-from-shading (SfS) principles. Following this approach, we introduce the following scattering distance

$$
d_{s c}(c, s)=\|\vartheta(c)-\vartheta(s)\|^{2}
$$

where $\vartheta(\cdot)$ is the local incidence angle.

\section{EXPERIMENTAL RESULTS}

The proposed non-local means SAR despeckling technique is applied to a Cosmo/SkyMed simulated SAR intensity image. A $39 \times 39$ search window is used; geometric distance is measured in pixels and incidence angles are measured in radians. Furthermore, the following parameter values are used: $\lambda_{\mathrm{g}}=0.003, \lambda_{\mathrm{sc}}=820, \lambda_{\mathrm{st}}=0.50$.

For simulation purposes we make use of SARAS, a SAR raw signal and image simulator [7]. The $512 \times 512$ simulated SAR image (Fig. 1(a)) has been synthesized starting from a fractal DEM with the Hurst exponent $H=0.8$ and the topothesy $T=10^{-4} \mathrm{~m}$. The electromagnetic parameters are the dielectric constant $\varepsilon_{r}=4$ and the electrical conductivity $\sigma$ $=0.01 \mathrm{~S} / \mathrm{m}$. Fig. 1(c) shows the despeckled SAR image using the proposed filter, while Figs. 1(d-e) show the output of the SAR block-matching 3-D (SAR-BM3D) [2] and the probabilistic patch-based (PPB) algorithm [3] respectively. For a quantitative assessment of the algorithms, a 512-look SAR image has been simulated and used as reference (Fig. 1(b)) and synthetic parameters have been evaluated, in particular the signal-to-noise ratio (SNR), despeckling gain (DG), mean value of image (MoI), mean of ratio (MoR), variance of ratio $(\mathrm{VoR})$, coefficient of variation $\left(\mathrm{C}_{\mathrm{x}}\right)$ and the mean value of structural similarity (MSSIM) index (Table I) (see [6] for definitions). From a visual inspection, the proposed despeckling filter provides great performances in terms of both speckle reduction and texture preservation as Fig. 1(d) shows, outperforming both SARBM3D and PPB. Thanks to the scattering distance term, pixels with very different incidence angles are not averaged although they are close to each other. A satisfactory details preservation is also reached. The performance parameters in Table I confirm the great accuracy provided by the proposed algorithm and clearly show in a quantitative way that performance comparable to the despeckling state of the art are reached.

\section{REFERENCES}

[1] A. Buades, B. Coll, and J. Morel, "A Review of Image Denoising Algorithms, with a New One," Multiscale Model. Simul., vol. 4, no. 2, pp. 490-530, 2005.

[2] S. Parrilli, M. Poderico, C.V. Angelino, and L. Verdoliva, "A Nonlocal SAR Image Denoising Algorithm Based on LLMMSE Wavelet Shrinkage," IEEE Trans. on Geosci. and Remote Sens., vol. 50, no. 2, pp. 606-616, Feb. 2012.

[3] C.-A. Deledalle, L. Denis, and F. Tupin, "Iterative Weighted Maximum Likelihood Denoising With Probabilistic Patch-Based Weights," IEEE Trans. Image Process., vol. 18, no. 12, pp. 26612672, Dec. 2009.

[4] L. Verdoliva, R. Gaetano, G. Ruello, and G. Poggi, "OpticalDriven Nonlocal SAR Despeckling," IEEE Geosci. and Remote Sens. Lett., vol. 12, no. 2, 314-318, 2015. 
[5] G. Di Martino, A. Di Simone, A. Iodice, D. Riccio, and G. Ruello, "On shape from Shading and SAR Images: an Overview and a New Perspective," in Proc. Int. Geosci. Remote Sens. Symp., pp. 1333-1336, 13-18 July 2014.

[6] G. Di Martino, M. Poderico, G. Poggi, D. Riccio, and L. Verdoliva, "Benchmarking Framework for SAR
Despeckling," IEEE Trans. on Geosci. and Remote Sens., vol. 52 , no. 3, pp. 1596-1615, Mar. 2014.

[7] G. Franceschetti, M. Migliaccio, D. Riccio, and G. Schirinzi, "SARAS: a synthetic aperture radar (SAR) raw signal simulator," IEEE Trans. on Geosci. and Remote Sens., vol. 30, no. 1, pp. 110123, Jan. 1992.

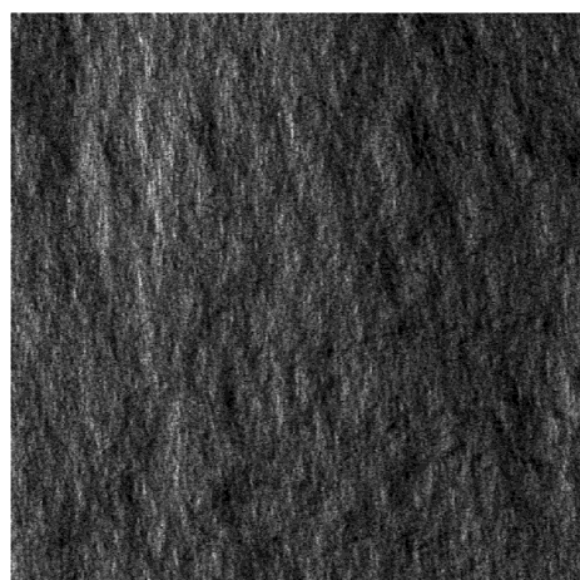

(a)

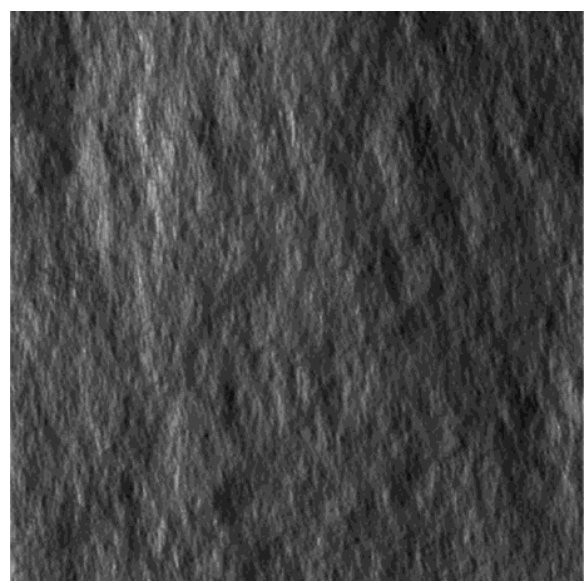

(d)

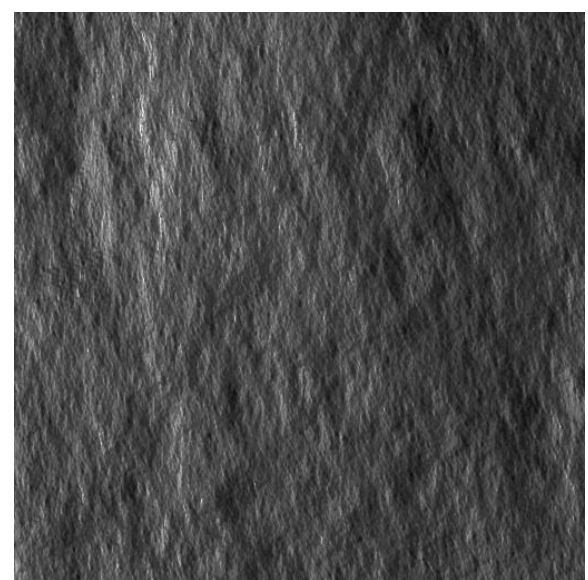

(b)

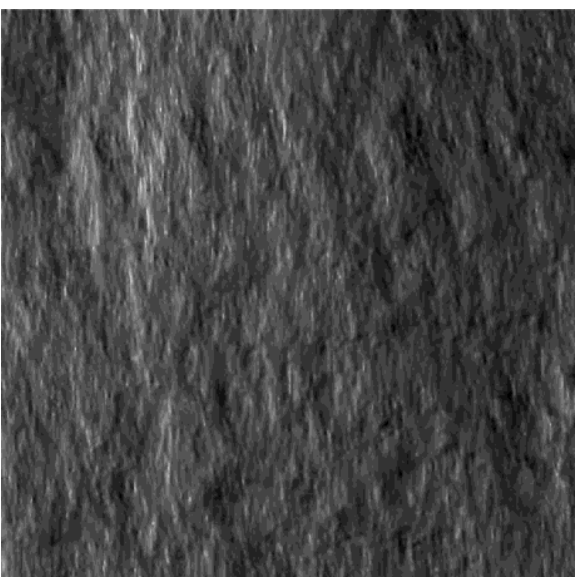

(e)

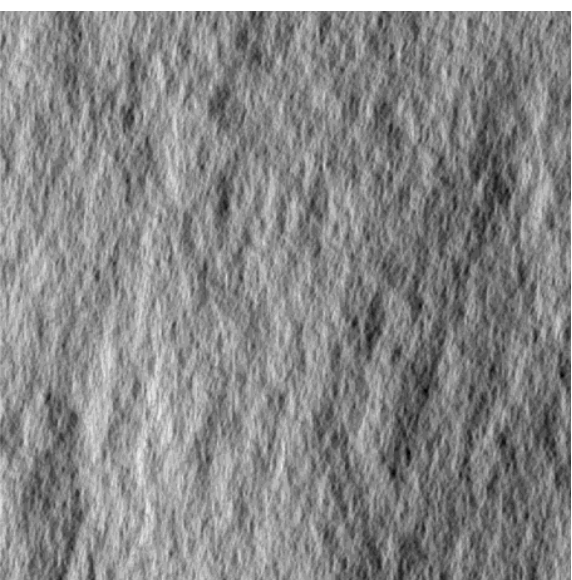

(c)

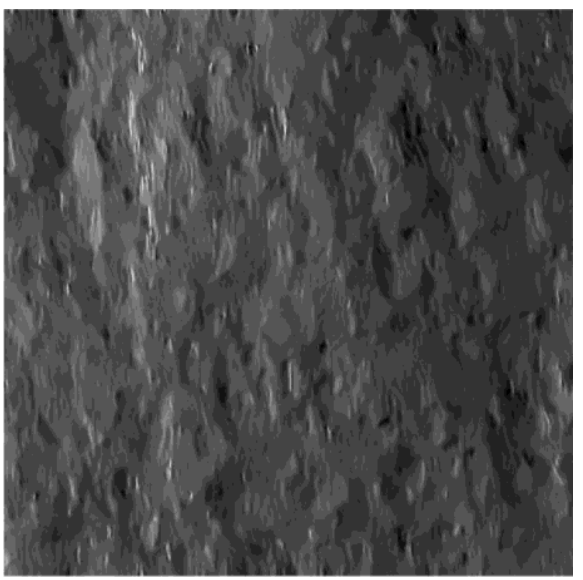

(f)

Fig. 1 (a) Single-look simulated SAR image of a 2-D fractal surface of parameters $H=0.8, T=10^{-4} \mathrm{~m}, \varepsilon r=4$ and $\sigma=0.01 \mathrm{~S} / \mathrm{m}$; (b) 512 look simulated SAR image used as reference; (c) Local incidence angle map in azimuth - slant range; (d) Proposed algorithm with $\lambda_{\mathrm{g}}=$ $0.003, \lambda_{\mathrm{sc}}=820, \lambda_{\mathrm{st}}=0.50$; (e) SAR-BM3D; (f) PPB.

TABLE I

PERFORMANCE PARAMETERS

\begin{tabular}{cccccccc}
\hline \hline & MoI & MoR & VoR & SNR & DG & $C_{x}$ & MSSIM \\
\hline Clean & 1.000 & 0.997 & 0.992 & $\infty$ & $\infty$ & 1.966 & 1.000 \\
Noisy & 0.991 & - & - & -0.559 & 0 & 2.955 & 0.963 \\
Proposed & 0.945 & 0.882 & 0.685 & $\mathbf{6 . 4 9 9}$ & $\mathbf{7 . 0 5 8}$ & 1.745 & $\mathbf{0 . 9 9 2}$ \\
SAR-BM3D & $\mathbf{0 . 9 4 9}$ & 0.867 & 0.545 & 4.919 & 5.478 & $\mathbf{1 . 9 0 4}$ & 0.986 \\
PPB & 0.948 & $\mathbf{0 . 8 8 8}$ & $\mathbf{1 . 0 5 3}$ & 4.324 & 4.883 & 1.686 & 0.980 \\
\hline \hline
\end{tabular}

